\title{
Efficient Iris Recognition Using Adaptive Quotient Thresholding
}

\author{
Peeranat Thoonsaengngam, Kittipol Horapong, \\ Somying Thainimit, and Vutipong Areekul \\ Kasetsart Signal and Image Processing Laboratory (KSIP lab), \\ Department of Electrical Engineering, Faculty of Engineering, \\ Kasetsart University, Bangkok, 10900, Thailand \\ $\{g 4765155$, g4565244, fengsyt, fengvpa\}@ku.ac.th
}

\begin{abstract}
This paper presents an intensity-based iris recognition system. The system exploits local intensity changes of the visible iris textures such as crypts and naevi. The textures are extracted using local histogram equalization and the proposed 'quotient thresholding' technique. The quotient thresholding partitions iris images in a database such that a ratio between foreground and background of each image is retained. By fixing this ratio, variations of illumination across iris images are compensated, resulting in informative and distinctive blob-like iris textures. An agreement of the two extracted textures is measured by finding spatial correspondences between the textures. The proposed system yields the $0.22 \%$ EER and 100\%CRR. The experimental results indicate encouraging and effective iris recognition system, especially when it is used in identification mode. The system is very robust to changes in decision ratio.
\end{abstract}

\section{Introduction}

The term 'biometrics' refers to the science and technology of authentication using physiological or behavioral characteristics of human such as fingerprints, signatures, faces, and irises. Among the biometrics, an iris pattern is a highly accurate and reliable characteristic. Flom and Safir [1] reported that the iris patterns are unique to individual and stable over time and across environments (e.g. occupations). The uniqueness of iris patterns is the product of dense collections of iris structures and textures such as pigment frills, furrows, freckles, and crypts. In addition, an iris is a protected organ located behind the cornea, but in front of the lens. This makes personal authentication possibilities life long.

Existing iris recognition systems were developed using several approaches. Major differences among the systems are methods used in analyzing and extracting iris features. Examples of these systems are briefly described as follows: Daugman [2] extracts iris textures using 2-D Gabor filter. Wides [3] analyses iris textures using 4-level Laplacian Pyramid. After these works, several iris recognition systems [4-5] have been proposed. However, few of them associate physical iris structures into their systems. One of the few is a system developed by Sun [6]. Sun applied zero-crossing wavelet transform to segment what he called blocks of interest-BOIs. From his experimental 
results, only partial physical iris structures are segmented. He, then, cascades the approach with a local feature based classifier to obtain higher recognition accuracy.

Our iris recognition system also attempts to segment the blob-like structures of an iris. However, the proposed system is a non-cascading system. Our approach exploits local intensity variations of the iris textures. The blob-like structures are extracted using local histogram equalization and the proposed technique, quotient thresholding. Our system consists of three main steps: preprocessing, feature extraction and feature matching. The following sections describe our system in details.

\section{Preprocessing}

The first step of our approach is to locate boundaries of an iris in an input eye image: the pupil's boundary and the sclera's boundary. These boundaries are approximated using concentric circles, in our approach.

The pupil boundary is detected by initially segmenting the pupil using thresholding technique. The pupil's boundary is located by applying edge detection, followed by edge thinning and circular fitting. The fitting yields the pupil's radius and its center. This center serves as the reference point for the rest of the process.

The sclera boundary is detected by exploiting intensity difference between iris and sclera. The contrast enhancement algorithm in [7] is firstly applied to improve contrast of the image. Then, average intensity of pixels along virtual arcs covered $\pm 45^{\circ}$ and moving outward from the center is calculated. The sclera boundary is located at the first abrupt change of the obtained average intensity.

Due to the iris size is varied depending on an amount of incoming light, the obtained iris images are normalized into the same fixed size.

\section{Our Proposed Feature Extraction}

Our feature extraction aims to extract visible and distinctive physical iris textures such as crypts, freckles, and moles. These textures occur randomly over the iris and have darker intensity level. They belong to regions of minimum of the image. Typically, the regions of minimum are segmented using conventional thresholding technique. One drawback of this technique is that it is sensitive to image illumination. Non-uniform illumination over and across images can significantly degrade its performance. Unfortunately, these non-uniformities can not be avoided during the iris acquisition process. In order to extract informative and discriminating iris textures, compensations for the non-uniform illumination must be accounted for. In this paper, we propose using local histogram equalization and quotient thresholding technique to compensate illumination changes over and across iris images.

\subsection{Local Histogram Equalization}

Local histogram equalization is used in our feature extraction in order to enhance iris textures and to compensate non-uniform illumination over the image. The local operation is chosen for two reasons. Firstly, local enhancement can compensate effects of non-uniform illumination of an image. Secondly, it is generally agreed that texture 
analysis should concern spatial distribution of pixels within a given neighborhood. This implies local operation is preferred over global operation for texture analysis.

Figure 1 illustrates effects of local versus global operation on segmenting the iris textures. The enhanced images of the same iris using global- and local- histogram equalization are shown in figure 1(a) and 1(c), respectively. Figure 1(b) and 1(d) show its corresponding segmented iris textures obtained by thresholding the enhanced images using threshold value of 150 , respectively. The obtained results reveal the global operation is unable to compensate the non-uniform illumination over the image. The global operation provides indistinguishable iris textures. Contrarily, the local histogram equalization provides informative and distinguishable iris textures.

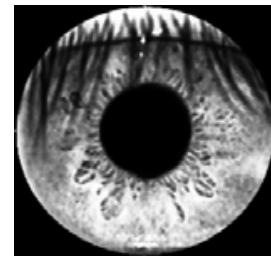

(a)

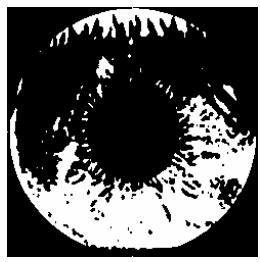

(b)

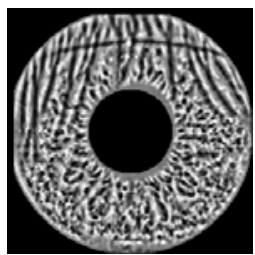

(c)

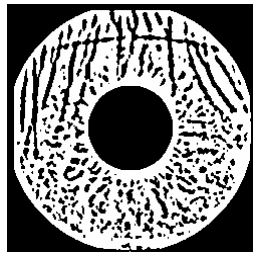

(d)

Fig. 1. (a) The enhanced iris image using global histogram equalization. (b) The segmented iris textures obtained by thresholding figure (a) using a threshold value of 150 . (c) The enhanced image of the same iris using local histogram equalization. (d) The segmented iris textures obtained by thresholding figure (c) using a threshold value of 150 .

From figure 1(d), does not only iris textures are enhanced, but also some irrelevant and unwanted textures. Since our approach utilizes local intensity changes of iris textures, noises caused from occlusions such as eyelashes can degrade our system performance dramatically. To reduce effects of these noises, only half radius of the iris ring covered $10^{\circ}$ above and $50^{\circ}$ below the iris's diameter is of our interest.

\subsection{Adaptive Quotient Thresholding}

In this paper, quotient thresholding is proposed to handle uneven illumination across iris images in a database. The motivation behinds the proposed thresholding is a human eye's adaptability characteristic. With our eye, a black-white pattern such as a checker lit differently is perceived similarly. If stationary scenes are assumed, this implies that a proportion between perceived black pixels and white pixels is the same. In term of image histogram, similar patterns can be achieved if a ratio between foreground and background is maintained. Maintaining this ratio is a principle of the proposed quotient thresholding technique.

The quotient thresholding partitions images in a database such that the ratio between foreground and background of each image, called decision ratio, is maintained. Figure 2 demonstrates our feature extraction scheme and its results. Two input images captured from the same iris are shown in the first row. The corresponding enhanced iris wedges using local histogram equalization overlaid over the iris ring are shown in the second row. The third row shows its corresponding histogram and the 

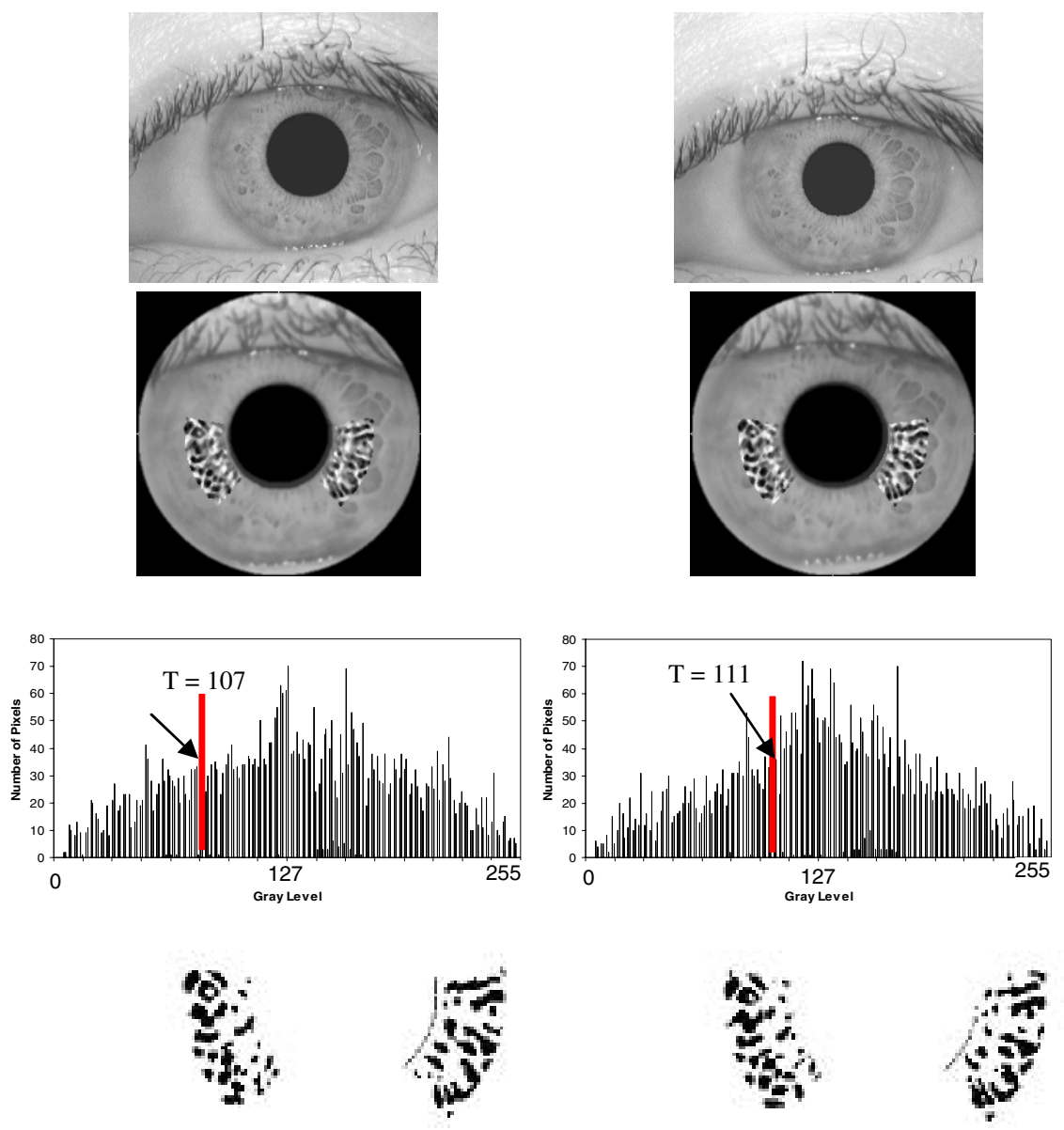

Fig. 2. The first row shows two images of the same iris. The second row shows corresponding enhanced iris wedges using local histogram equalization overlaid over the iris ring. The third row shows corresponding image histograms with the threshold value indicators. These threshold values yield 0.33 decision ratio. The last row displays corresponding segmented iris textures using the threshold values.

threshold value used to obtain a 0.33 decision ratio. The thresholded textures are displayed in the last row. It is clearly seen that the obtained textures well reflect the physical iris structures. Though the proposed feature extraction is very simple, it is very effective.

\section{Iris Feature Matching}

An agreement of two extracted iris textures is measured by finding spatial correspondences between the textures. The number of aligned pixels is counted and reflected in 
term of a matching score. In our system, the rotation and translation invariance is achieved by rotating and translating the template within a range of $\pm 10^{\circ}$ and \pm 10 pixels, respectively. A matching score of each pair is calculated. The maximum matching score among them represents the matching score of the template.

\section{Experimental Results}

We validate our proposed iris recognition using CASIA iris database [8]. The database consists of 108 irises. Each iris has 7 images. The recognition system is implemented using VC++ on PC Pentium IV 2.4 GHz with $512 \mathrm{MB}$ memory.

Table 1. Computational time of the proposed iris recognition system

\begin{tabular}{|l|c|}
\hline Process & Computational Time (msec.) \\
\hline Preprocessing & 370 \\
\hline Feature Extraction & 250 \\
\hline Feature Encoding & 20 \\
\hline Feature Matching & 17 \\
\hline
\end{tabular}

Table 2. Our proposed system performance

\begin{tabular}{|c|c|c|}
\hline $\begin{array}{l}\text { Decision } \\
\text { Ratio }\end{array}$ & EER (\%) & CCR (\%) \\
\hline 0.11 & 0.416487 & 100 \\
\hline 0.13 & 0.344492 & 100 \\
\hline 0.15 & 0.290305 & 100 \\
\hline 0.17 & 0.293837 & 100 \\
\hline 0.19 & 0.291188 & 100 \\
\hline 0.21 & 0.288009 & 100 \\
\hline 0.23 & 0.306199 & 100 \\
\hline 0.25 & 0.297722 & 100 \\
\hline
\end{tabular}

\begin{tabular}{|c|c|c|}
\hline $\begin{array}{l}\text { Decision } \\
\text { Ratio }\end{array}$ & EER (\%) & CCR (\%) \\
\hline 0.27 & 0.260135 & 100 \\
\hline 0.29 & 0.244447 & 100 \\
\hline 0.31 & 0.259605 & 100 \\
\hline 0.33 & 0.219723 & 100 \\
\hline 0.35 & 0.264374 & 100 \\
\hline 0.37 & 0.251335 & 100 \\
\hline 0.39 & 0.342196 & 99.8677 \\
\hline 0.40 & 0.348024 & 99.8677 \\
\hline
\end{tabular}

Our first experiment is finding the decision ratio offered the best system performance. The system performance is measured in terms of Equal-Error-Rate (EER) and Correct-Recognition-Rate (CRR) using the leave-two-out method. Two images out of 756 images are randomly selected as test images. Table 1 indicates computational time of our system. Table 2 indicates the obtained EER and CRR for each decision ratio. From the experiment, decision ratio of 0.33 yields the best system performance with $0.22 \%$ EER and $100 \%$ CRR. From the results, our system is very well performed in an identification mode. Almost all decision ratios yield the $100 \%$ CRR. In addition, the results indicate that our system is quite insensitive to values of decision ratio.

Next, we examine the distribution of intra- and inter- class verification. The distribution of the matching distance is shown in figure 3. Comparing to existing iris 


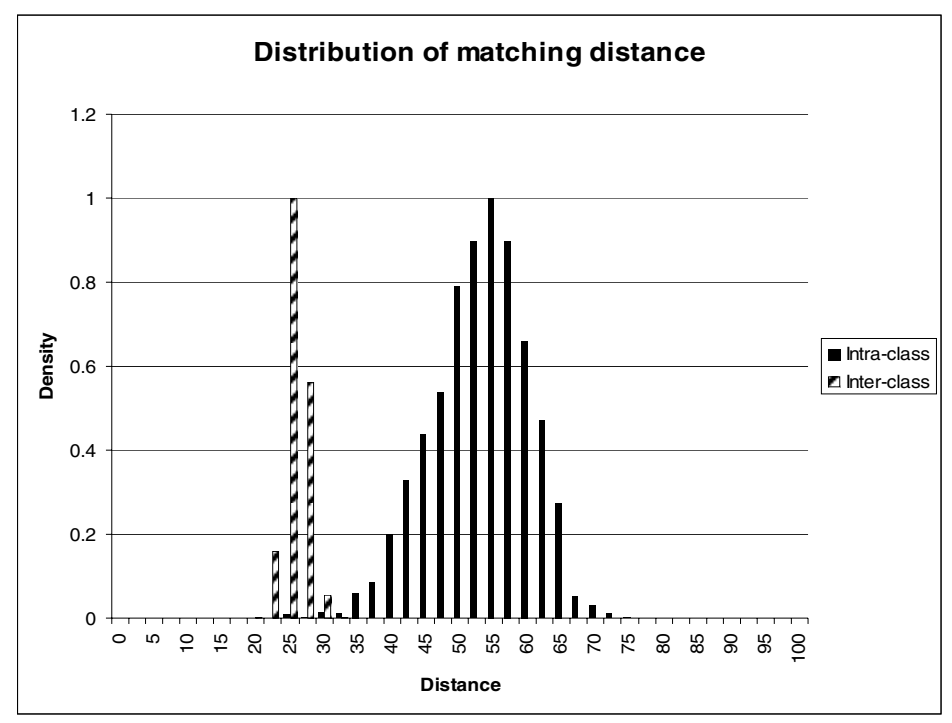

Fig. 3. The distribution of inter- and intra- class verification using 0.33 decision ratio

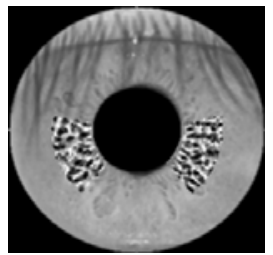

(a)

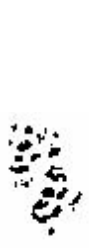

(b)

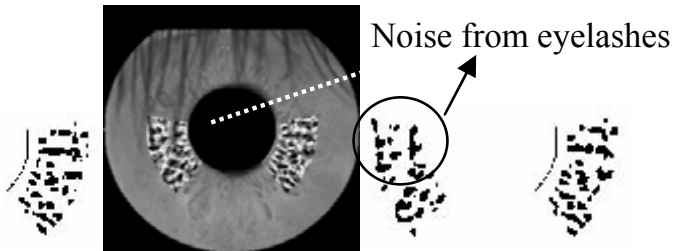

(c)

(d)

Fig. 4. (a) An iris image overlaid by its enhanced iris textures. (b) Its corresponding extracted textures. (c) Another image of the same iris captured during eyelashes are moving downward. (d) Its corresponding extracted textures. The circle in the figure indicates areas where the occlusions occur.

recognition systems [2-6], the proposed iris system performance in verification mode is lower. From our observations, main degradations of our system are caused from occlusion noises such as eyelashes. Since eyelashes have very low intensity, they are always included in the segmented textures. As a result, the segmented textures appear differently where the occlusions occur as shown in figure 4. Further system improvement can be achieved by integrating eyelids and eyelashes removal scheme into our system.

\section{Conclusions and Future Works}

An efficient intensity-based iris recognition algorithm is proposed in this paper. The local intensity variation of physical iris textures is exploited in the proposed method. 
The physical iris textures are analyzed and extracted using local histogram equalization and adaptive quotient thresholding. The quotient thresholding partitions an image in a database into foreground and background such that a ratio between the foreground and background of all images in the database are maintained. The proposed algorithm yields informative and discriminating blob-like iris features. The matching between the input feature and the template is measured by finding spatial correspondences between the features. Our experimental results show encouraging iris recognition performance. With 0.33 decision ratio, our system yields the $0.22 \%$ EER and $100 \%$ CRR. Hence, the proposed system is very efficient but very simple.

\section{Acknowledgement}

This work was partially supported by the National Electronics and Computer Technology Center (NECTEC) under National Science and Technology Development Agency (NSTDA) under Grant NT-B-22-I3-12-47-07.

\section{References}

1. L. Flom, A. Safir,: Iris Recognition System. U.S. Patent No. 4641 349, 1987.

2. J.Daugman: Biometric Personal Identification System based on Iris Analysis. U.S. Patent No. 5291 560, 1994.

3. R. P. Wildes: Iris Recognition: An Emerging Biometric Technology. Proceeding of the IEEE, Vol. 85, No.9, pp.1348-1362, 1997.

4. W.W. Boles and B.Boashah: A Human Identification Technique using Images of the Iris and Wavelet Transform. IEEE Trans. on Signal Processing, Vol.46, pp1185-1188, 1998.

5. Lima, Tieniu Tan, Yunhong Wang, Dexie Zhang: Personal Identification Based on Iris Texture Analysis. IEEE Trans. on PAMI, Vol. 25, No.12, pp.1519-1533, 2003.

6. Zhenan Sun, Yunhong Wand, Tieniu Tan, and Jiali Cui: Cascading Statistical and Structural Classifiers for Iris Recognition. Proceedings of ICIP, pp.1261-1264, 2004.

7. L.Hong, Y.Wan, and A.K.Jain: Fingerprint Image Enhancement: Algorithm and Performance Evaluation. IEEE Trans. on PAMI, Vol. 20, No.8, pp.777-789, 1998.

8. http://www.sinobiometrics.com. 